\title{
A Single-Central, Randomized, Double-Blinded, Placebo-Controlled, Crossover Trial Protocol: A Clinical Effect Evaluation Study on the TCM Comprehensive Intervention Program for Chronic Heart Failure
}

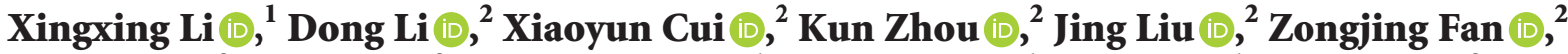

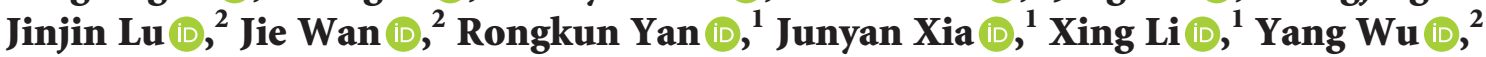 \\ Qian Lin $\left(D^{3},{ }^{3}\right.$ and $\operatorname{Yan} \operatorname{Li} \oplus^{2}$ \\ ${ }^{1}$ Department of Graduate School, Beijing University of Chinese Medicine, Beijing 100029, China \\ ${ }^{2}$ Department of Cardiology, Dongfang Hospital, Beijing University of Chinese Medicine, Beijing 100078, China \\ ${ }^{3}$ Department of Cardiology, Dongzhimen Hospital, Beijing University of Chinese Medicine, Beijing 100700, China
}

Correspondence should be addressed to Qian Lin; linqian62@126.com and Yan Li; 15801434320@163.com

Received 5 June 2021; Accepted 2 December 2021; Published 28 December 2021

Academic Editor: Youhua Wang

Copyright (C) 2021 Xingxing Li et al. This is an open access article distributed under the Creative Commons Attribution License, which permits unrestricted use, distribution, and reproduction in any medium, provided the original work is properly cited.

\begin{abstract}
Background. It is known to all the doctors and patients that both the morality and incidence rate of chronic heart failure (CHF) are quite high among various heart diseases. Traditional Chinese medicine (TCM) comprehensive intervention becomes a rising prospective therapy for patients with CHF. Considering the efficacy of TCM, the study aims to test the safety as well as the validity of TCM comprehensive intervention in patients who are struggling with CHF. Methods. The study is an essentially randomized, single-central, placebo-controlled, double-blinded crossover trial. Eighty-two eligible subjects aged 18-75 years with CHF are supposed to be recruited. According to the subject plan, all the patients will be divided into group A and B. The patients in group A will receive oral Qishen Taohong granules (QTGs) combined with TCM external treatment and standard Western medicine for four weeks. After that, a 2-week washout would be proceeded; this group will be reallocated to another four weeks with oral placebo granules combined with TCM external treatment and standard Western medicine. In contrast, group B will perform the opposite protocol. The primary outcome conforms to the classification from the New York Heart Association (NYHA). Meanwhile, the secondary outcomes are echocardiogram, N-terminal pro-B-type natriuretic peptide (NT-proBNP), Chronic Heart Failure Quality of Life Scale of Integrated Chinese and Western Medicine (CHFQLS), TCM syndrome, symptom, sign, six-minute walk test (6MWT), Pittsburgh Sleep Quality Index (PSQI), Montreal cognitive assessment (MoCA), major adverse cardiovascular events (MACEs), and metabolomics. Discussion. Based on conventional treatments, TCM comprehensive intervention may further improve the patients' cardiac function and then enhance their quality of life. The results will provide high-quality evidence of TCM comprehensive intervention in treating CHF.
\end{abstract}

\section{Introduction}

Chronic heart failure (CHF) is the final form of cardiovascular diseases [1]. It could be fatal, disabling, and costly and has become an increasingly important global health problem [2]. CHF patients usually experience a malignant disease cycle: "hospitalization-improvement-discharge-rehospitalization" [3]. Epidemiological studies have shown that about 64.3 million people in the whole world are suffering different kinds of heart failure [4]. Recently, a meta-analysis was conducted with more than 1.5 million patients who have heart failure. The result shows that 1-, 2-, 5-, and 10-year survival rates, respectively, increase to $87 \%, 73 \%, 57 \%$, and $35 \%$ [5]. Despite advances in the treatment strategies over the past 30 years, the prognosis of patients remains poor and the quality of life (QOL) remains impaired [6].

Thus, novel effective and safe treatment options for these patients are highly wanted. Chinese herbal 
medicine (CHM), especially combined herbal prescription and external treatment, is widely applied in traditional Chinese medicine (TCM) to treat CHF, and in recent years, more and more randomized controlled trials (RCTs) have confirmed its advantages in CHF treatment [7-9]. The Chinese herbal prescription, Qishen Taohong granules (QTGs), is composed of nine herbal drugs that can nourish Qi, disperse blood stasis, and remove water. In our previous studies, QTGs had a good effect in improving the cardiac function while enhancing the QOL of CHF patients $[10,11]$. The positive role of TCM external treatment on CHF had also been confirmed [7]. We propose that CHF patients treated with QTG combined with TCM external treatment will have more positive clinical outcomes than patients receiving the control drug.

However, to the best of our knowledge, reliable evidence-based medical data have not yet been reported for the treatment of CHF with TCM comprehensive interventions (CHM combined with external treatment). Within this context, a placebo-controlled, double-blind, and randomized crossover trial will be carried out to assess the efficacy of QTG combined with TCM external treatment in treating CHF.

\section{Methods and Analysis}

2.1. Design. This is a single-center, prospective, randomized, placebo-controlled, double-blind, crossover trial. Also, this trial has been formally enrolled at https://www.chictr.org.cn (ChiCTR2000038737). This study protocol promised the ethical requirements according to the 1975 Declaration of Helsinki. Meanwhile, this research has been permitted by our institutional review broad (JDF-IRB-2020031002), and the study flow is shown in Figure 1.

\subsection{Participants}

2.2.1. Diagnostic Criteria. In order to ensure the scientific validity of the study, Framingham heart failure criteria are employed to diagnose CHF [12]. The reference of cardiac function staging originates from the American Heart Association (AHA) CHF staging [13]. The base of cardiac function grading lies in the grading protocol established by the former association NYHA [14]. TCM syndrome is identified by the treatment of heart failure as it writes "New drug investigation research guidelines for traditional Chinese medicine [15]".

2.2.2. Inclusion Criteria. The qualified subjects of this trial need to fulfill the following standards: (1) age between 18 and 75 years, (2) men and women who have been diagnosed as CHF, on the stage C of NYHA grade II or III, (3) TCM syndrome in forms of differentiation Qi deficiency, blood stasis, and water retention, (4) left ventricular ejection fraction $(\mathrm{LVEF})<40 \%$ or $40 \% \leq \mathrm{LVEF} \leq 50 \%$ and NT-proBNP $>300 \mathrm{ng} / \mathrm{L}$, and (5) provision of written informed consent.
2.2.3. Exclusion Criteria. Subjects will be excluded if they had (1) acute myocardial infarction, (2) difficulties with walking, (3) severe mental dysfunction, pregnancy, or any malignancy, (4) severe liver and/or renal dysfunction, (5) severe anemia, and (6) taken TCM (including Chinese patent medicine or TCM injection or Chinese herbal medicine) or participated in other trials within 2 weeks.

2.2.4. Termination Criteria. The termination criteria are as follows: (1) those who do not meet the inclusion criteria but are mistakenly enrolled, (2) those who are failing to follow the study protocol, (3) those who are having serious drug allergies or adverse reactions, and (4) various other reasons such as the patients who were asked to withdraw before the trial ended.

2.3. Randomization and Masking. Subjects will be divided into group A and B by the ratio of $1: 1$ by using sequentially numbered, sealed, opaque envelopes. The grouping and sequence will be summarized and stored on a computer by an independent statistician with no knowledge of the study design.

2.4. Blinding. Double blinding will be adopted in this study. Patients, investigators, outcome evaluators, and data analysts will all be unaware of treatment allocation throughout the study period. In terms of appearance, texture, color, and taste, placebo granules and QTGs are quite similar. At the end of this study, the emergency envelope will be assigned for every drug number, which represented the group information of the drug. The envelopes could only be opened when medical emergencies occur. In addition, once the emergency envelope is opened, the operator will be asked to sign and enter the date in the margins on the cover of letters, as well as the reasons of opening. After all the research data had been collected and recorded, the unblinding process will be carried out.

2.5. Intervention. Group A subjects will be given oral QTG combined with TCM external treatment and standard Western medicine for 4 weeks. Following a 2-week washout, patients in group A will input oral placebo granules combined with TCM external treatment and standard Western medicine for 4 weeks. Group B patients will be involved in the opposite medical procedure. During the washout period, patients will only receive standard Western medicine.

2.5.1. Standard Western Medicine. The standard Western medicine follows the guidelines for CHF treatment [16]: (1) angiotensin-converting enzyme inhibitor (ACEI) or angiotensin II receptor blocker (ARB) or angiotensin receptor neprilysin inhibitors (ARNIs): fosinopril $10 \mathrm{mg}$ once a day or valsartan capsule $80 \mathrm{mg}$ once a day or other similar drugs, (2) beta-blockers $(\beta-B)$ : metoprolol succinate $47.5-190 \mathrm{mg}$ once a day or bisoprolol fumarate 5-10 mg once a day, (3) diuretics: hydrochlorothiazide $12.5-25 \mathrm{mg}$ once a day or furosemide $20 \mathrm{mg}$ once or twice a day or tolasamide $20 \mathrm{mg}$ 


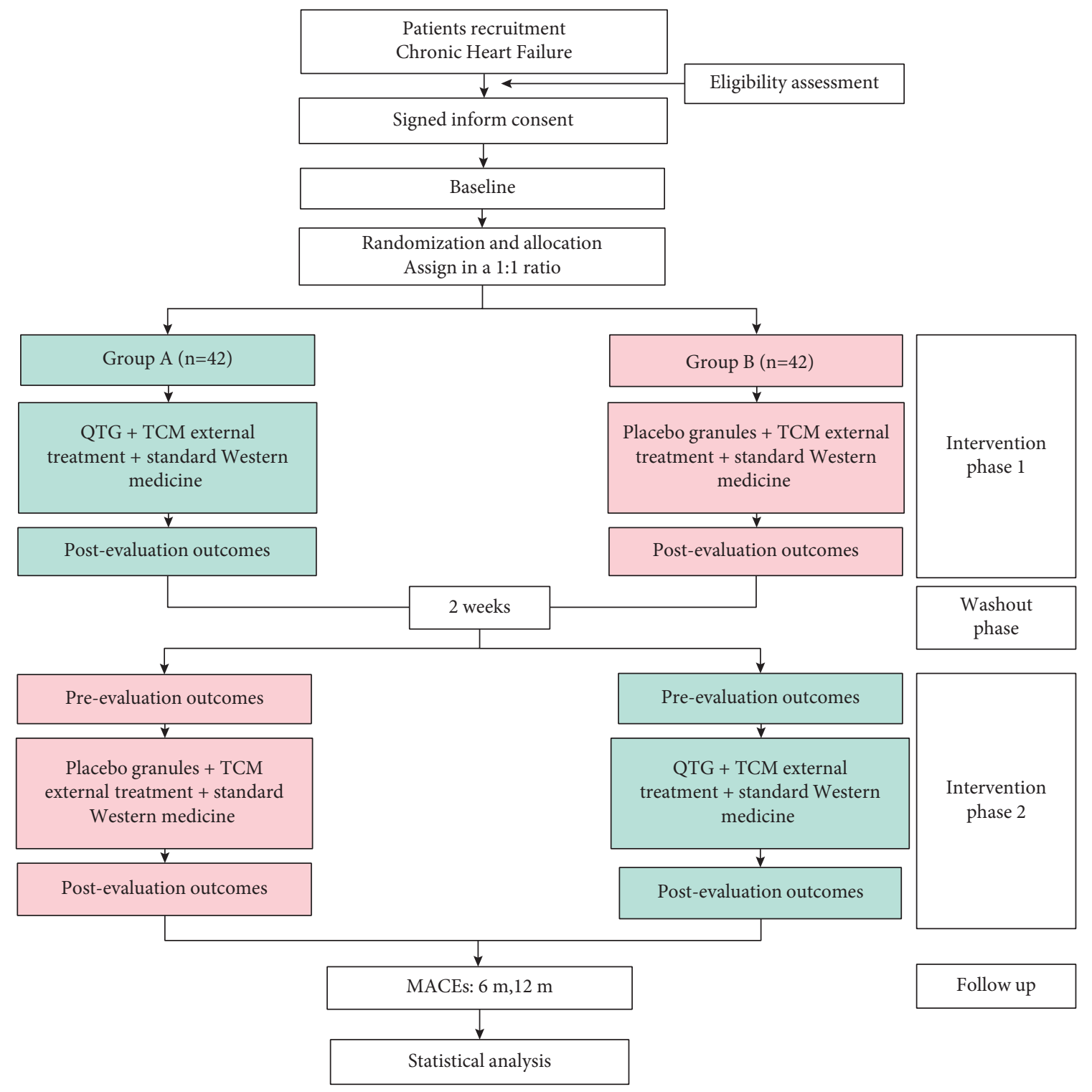

Figure 1: Study flow diagram.

once or twice a day (combined with $1 \mathrm{~g}$ potassium chloride sustained release tablets once to three times a day, depending on the patient's condition), (4) isosorbide dinitrate: aldosterone receptor antagonist: spironolactone $20 \mathrm{mg}$ once a day, and (5) digoxin once a day if necessary.

\subsubsection{TCM External Treatment. (1) Acupoint Sticking} Therapy [17]. The acupoint sticking therapy mainly consisted of Salvia miltiorrhiza $10 \mathrm{~g}$, cassia twig $10 \mathrm{~g}$, Ligusticum wallichii $10 \mathrm{~g}$, and Pericarpium trichosanthis $10 \mathrm{~g}$, and the ointment of acupoint sticking will be applied with patching time $4 \mathrm{~h}$ of each and once every three days. The acupuncture points will be selected are shenque, mingmen, and danzhong. Acupoint application for all patients will be performed by the same trained nurse.

(2) Tongue Exercise [18]. The patient will do the following steps once a day: licking the upper jaw 30 times, licking cheeks 4 times, licking gums 8 times, licking the corners of the mouth 10 times, slapping the tongue 20 times, and stretching the tongue 10 times. They would also be required to complete the tongue exercise, under the guidance of the same trained nurse.

(3) Auricular Point Acupressure [19]. The specific ear points that will be treated are the heart, shenmen and sympathesis, chest, and other points. After ear skin was disinfected with alcohol cotton balls, $0.5 \mathrm{~cm} \times 0.5 \mathrm{~cm}$ square tapes with the seeds of cowherb will be pressed on the established ear points until the patients experience a sense of acid distention (de qi), 5 times a day, with 2 min time of each. The seeds of cowherb will be replaced weekly. The same welltrained nurse performed all auricular point acupressure.

2.5.3. QTGs. QTGs were obtained from Beijing Kangrentang Pharmaceutical Co., Ltd. (Beijing, China). The composition of the prescription is as follows: 60 grams (g) of Astragalus membranaceus, $15 \mathrm{~g}$ of Codonopsis pilosula, $15 \mathrm{~g}$ 
of Salvia miltiorrhiza, $10 \mathrm{~g}$ of Semen persicae, $10 \mathrm{~g}$ of Carthamus tinctorius, $10 \mathrm{~g}$ of Cortex mori, $15 \mathrm{~g}$ of Semen lepidii, $15 \mathrm{~g}$ of Polyporus umbellatus, and $15 \mathrm{~g}$ of Lycopus lucidus. Patients in phase 1 of group A and phase 2 of group B will receive QTG $11.2 \mathrm{~g}$ (brewed with $100-200 \mathrm{ml}$ water) twice a day for 4 weeks.

2.5.4. Placebo. The placebo of QTGs that was made up of dextrin, a bitter agent, and 5\% dose of the QTG composition was also prepared by the same company. It is similar to the QTGs in terms of appearance, taste, and smell. Patients in phase 2 of group A and phase 1 of group B will receive placebo granules $11.2 \mathrm{~g}$ (brewed with $100-200 \mathrm{ml}$ water) twice a day.

\subsection{Outcomes}

2.6.1. Primary Outcome. The primary outcome is NYHA classification [15]. The NYHA classification is increased by at least 2 to be markedly effective, increased by 1 to be effective, increased by less than 1 to be ineffective, and reduced by at least 1 to be deteriorating.

2.6.2. Secondary Outcomes. The following secondary outcomes will be marked before treatment and after treatment: (1) echocardiogram, (2) N-terminal pro-B-type natriuretic peptide (NT-proBNP), (3) Chronic Heart Failure Quality of Life Scale of Integrated Chinese and Western Medicine (CHFQLS) [20], (4) TCM syndrome score, (5) symptom score, (6) sign score, (7) six-minute walk test (6MWT), (8) Pittsburgh Sleep Quality Index (PSQI), (9) Montreal cognitive assessment (MoCA), (10) major adverse cardiovascular events (MACEs), and (11) metabolomics.

2.6.3. Safety Outcomes. The safety assessment is based on vital signs, laboratory tests, and adverse reactions (ARs). Vital signs could be observed by breathing, blood pressure, heart rate, and temperature. Laboratory tests are mainly blood routine, renal function, liver function, urine routine, and serum electrolytes. The research team will specifically record the abnormal events during the study period.

If serious ARs occur, the researcher should immediately report to the ethics committee and take timely treatment measures. The schedule of intervention and assessments is shown in Table 1.

2.7. Sample Size Estimation. Estimating the effective rate according to the classification of NYHA, the sample size has been figured out. Based on previous study results, the effective rate in the QTG group was $84 \%$, while the effective rate of the control group is around $60 \%$ [11]. According to the formula $n 1=n 2=2^{-} \mathrm{p}^{-} \mathrm{q}\left(Z_{\alpha}+Z_{\beta}\right)^{2} /(\mathrm{p} 1-\mathrm{p} 2)^{2}=74$, the result is $n=2 \times 74=148$ subjects. Also, the sample size for the crossover intervention trial is generally half that of the randomized controlled trial under the same situation [22]. Moreover, because the dropout rate is about $10 \%, 82$ subjects are supposed to be randomly accounted to ensure the prescribed number of patients.

2.8. Data Collection and Management. The case record form (CRF) will be applied to the collection of relevant medical records. The CRF will be reviewed by clinical investigators and supervisors to be checked. Data were recorded in MS Excel 2007. For the data accuracy, two administrators will intervene and proofread independently. After the review and confirmation, the database will be locked. In principle, the locked data file cannot be modified again. If errors will be found, they will be corrected in the process of statistical analysis after confirmed, recorded, and explained.

2.9. Statistical Analysis. Intention-to-treat (ITT) and perprotocol (PP) analysis would be planned while the same results indicate that the evaluation results are reliable. All the randomized data would be analyzed by the ITT analysis, and missed data due to withdrawal will be finally substituted by the last observation carried forward (LOCF) or by the intragroup mean of the variable. If necessary, the missing value processing method provided by SPSS software will be adopted. The PP dataset will be used to analyze patients with good adherence to the research protocol.

Continuous data are represented by mean \pm standard deviation (SD), and categorical data are represented by the degree of frequency. Considering the normal distributed continuous variable items, the independent $t$-test will be used for comparison between group A and group B, and the paired $t$-test will be used for comparison within each group; otherwise, a nonparametric test will be taken into consideration. Categorical variables will be analyzed with the help of Wilcoxon's test and the chi-squared test. For the correlation analysis of the two continuous data, when the data meet normality, Pearson correlation analysis will be used; otherwise, Spearman rank correlation analysis will be used.

$P<0.05$ means that there is huge difference in the statistics. All data will be analyzed using the Statistical Product and Service Solutions (SPSS) unless otherwise noted.

2.10. Monitoring. Monitoring will be conducted by two members of the clinical research centre independent of the study group. Study visits will occur throughout the conduct of the study at least once each year. When nonconcordance occurred, a report will be immediately given to the principal investigator, and a second visit will be scheduled.

\section{Discussion}

Our study is the first double-blinded, randomized, placebocontrolled trial to investigate whether the TCM comprehensive intervention (QTGs combined with TCM external treatment) has a greater effect in patients with CHF.

CHF is a complex condition, with high morbidity, mortality, and treatment costs [23]. The current treatment of CHF has limitations [24]. Complementary and alternative therapies are, therefore, required in some patients with CHF. 
TABLE1: Schedule of intervention and assessments.

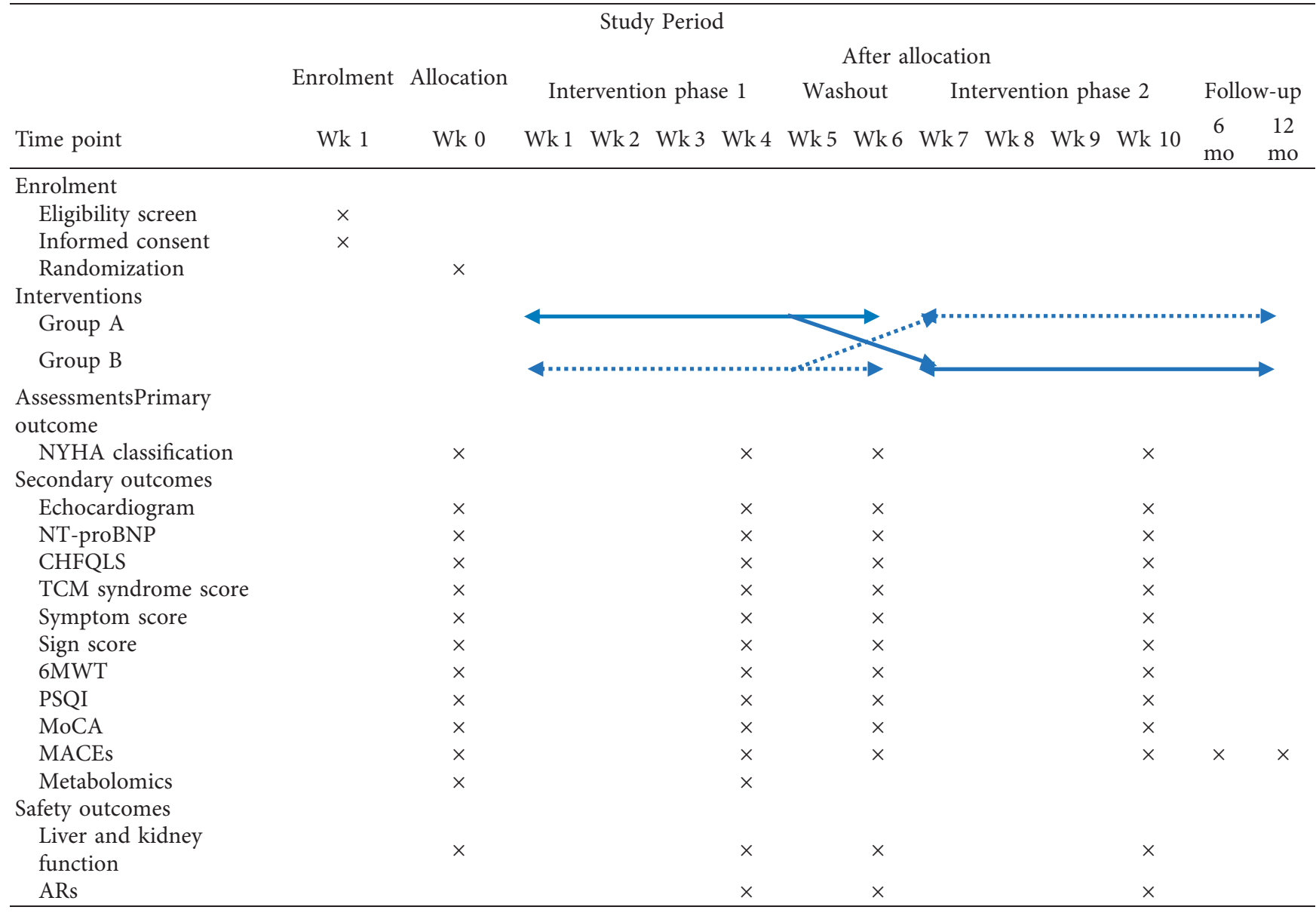

Schedule of enrollment, intervention, and assessment for the MIRACLE trial was according to the Standard Protocol Items: Recommendations for Interventional Trials (SPIRIT) [21]. Wk, week; mo, month.

Evidence about the effectiveness of TCM comprehensive interventions for CHF patients is also limited. The study results are poorly comparable and have little scientific proof, and it is difficult to adequately illustrate the advantages of TCM.

Several clinical studies have shown that TCM improves the clinical symptoms, cardiac function, and QOL in people with CHF [25-27]. However, there are still few studies on the high level of evidence in this field, and most of them are based on Chinese herbal medicine. In the meantime, tongue exercise, acupoint sticking therapy, auricular point acupressure, and other TCM external treatments are also playing a significant role in clinics. It also fully embodies the characteristics of being simple, convenient, cheap, and effective in TCM, which is easy to master and popularize. The TCM comprehensive intervention can break through the limitation of the current TCM treatment program for CHF by using only prescriptions so that a large number of $\mathrm{CHF}$ patients distributed in Western medicine hospitals and primary hospitals can also benefit from it, and it has broad application prospects. Therefore, TCM comprehensive intervention is a potential feasible candidate for the $\mathrm{CHF}$ treatment.

QTGs are a TCM prescription with good clinical effects. Our previous studies have shown that QTGs could enhance CHF patients' cardiac function, TCM syndrome score, and
QOL of patients with CHF $[10,11]$. They also display a cardioprotective effect in animal models of heart failure and prevent left ventricular remodeling [28]. However, the effect of QTGs combined with TCM external treatment on CHF still requires confirmation.

Our study has strengths to a certain degree. Compared to the previous study, it is the first double-blind, placebo-controlled crossover study to assess the validity as well as the safety of TCM comprehensive intervention in CHF treatment. A crossover trial is a good choice in clinical studies because it avoids differences between groups and eliminates differences between individuals, thereby improving the accuracy of efficacy evaluation by evaluating the results in different medical interventions for each patient [29]. Also, the required sample size of a crossover trial is smaller than that of an RCT, which better solves the problem of limited number of subjects and improves the evidence-based medicine evidence for the treatment of $\mathrm{CHF}$ with TCM comprehensive intervention [30]. These will make up the shortcomings of the previous studies and make potential results generalizable.

However, this study also has some limitations. First, it lacks new oral drugs (such as Sacubitril-Valsartan and SGLT-2i) for the treatment of CHF as a positive drug. In addition, this study will be performed in China, and it is 
uncertain whether other ethnic groups and regions can achieve similar effects.

Thus, due to the lack of similar research, we believe that the performance of our study and publication of the results will provide scientific support for the use of TCM comprehensive intervention in individuals with $\mathrm{CHF}$ to improve their cardiac function and QOL.

\section{Abbreviations}

CHF: $\quad$ Chronic heart failure

TCM: Traditional Chinese medicine

QTGs: Qishen taohong granules

NYHA: $\quad$ New York Heart Association

NT- N-terminal pro-B-type natriuretic peptide

proBNP:

CHFQLS: Chronic Heart Failure Quality of Life Scale of Integrated Chinese and Western Medicine

MoCA: Montreal cognitive assessment

MACEs: Major adverse cardiovascular events

QOL: Quality of life

CHM: $\quad$ Chinese herbal medicine

RCTs: $\quad$ Randomized controlled trials

AHA: American Heart Association

ACEI: Angiotensin-converting enzyme inhibitor

ARB: Angiotensin II receptor blocker

ARNI: Angiotensin receptor neprilysin inhibitors

$\beta$-B: $\quad$ Beta-blockers

LVEF: Left ventricular ejection fraction

6MWT: Six-minute walk test

PSQI: $\quad$ Pittsburgh Sleep Quality Index

Wk: Week

mo: $\quad$ Month

ARs: $\quad$ Adverse reactions

CRF: $\quad$ Case record form

ITT: Intention-to-treat

PP: $\quad$ Per-protocol

LOCF: Last observation carried forward

SD: $\quad$ Standard deviation

SPSS: $\quad$ Statistical Product and Service Solutions.

\section{Data Availability}

The study is currently in the stage of recruitment of participants. The results of this study along with the full intervention protocol and participants' data will be published upon completion.

\section{Ethical Approval}

This study has obtained the approval of the Ethics Committee of Dongfang Hospital affiliated with Beijing University of Chinese Medicine (JDF-IRB-2020031002). The implementation of this study adhered to the guidelines of the Declaration of Helsinki and Tokyo for humans.

\section{Consent}

Written consent has been obtained from all the patients involved.

\section{Disclosure}

The study funders have no role in the study design, data collection and management, and manuscript writing.

\section{Conflicts of Interest}

The authors declare no conflicts of interest.

\section{Authors' Contributions}

LY and LQ contributed to the design of the study and registration of the trial. LX drafted the manuscript. LY, LQ, and WY contributed to the revision of the manuscript. LX, LD, CX, LJ, FZ, LJ, YR, XJ, and LX contributed to the execution of the study. In addition, $\mathrm{ZK}$ and WJ formulated the methodology. All the authors reviewed the final manuscript and gave approval for the publication of this protocol.

\section{Acknowledgments}

This study was inspired and endorsed by the grant of the Capital Health Development Research Project (grant no. 2020-2-4201) (major funding body), National Natural Science Foundation of China (grant no. 81973622), and Beijing University of Chinese Medicine 1166 Development Program for Junior Scientists (grant no. 030903010331).

\section{Supplementary Materials}

Additional file 1: Completed Standard Protocol Items: Recommendation for Interventional Trials (SPIRIT) 2013 Checklist: items addressed in this clinical trial protocol. Additional file 2: informed consent form. (Supplementary Materials)

\section{References}

[1] T. A. McDonagh, M. Metra, and M. Adamo, "ESC Guidelines for the diagnosis and treatment of acute and chronic heart failure," European Heart Journal, vol. 42, no. 36, pp. 3599-3726, 2021.

[2] Y. Rachamin, R. Meier, T. Rosemann, A. J. Flammer, and C. Chmiel, "Heart failure epidemiology and treatment in primary care: a retrospective cross-sectional study," ESC Heart Failure, vol. 8, no. 1, pp. 489-497, 2021.

[3] G. Savarese, N. Sattar, J. Januzzi et al., "Empagliflozin is associated with a lower risk of post-acute heart failure rehospitalization and mortality," Circulation, vol. 139, no. 11, pp. 1458-1460, 2019.

[4] Collaborators GDaIIaP, "Global, regional, and national incidence, prevalence, and years lived with disability for 354 diseases and injuries for 195 countries and territories, 1990-2017: a systematic analysis for the global burden of disease study 2017," Lancet, vol. 392, no. 10159, pp. 1789-1858, 2018.

[5] N. R. Jones, A. K. Roalfe, I. Adoki, F. D. R. Hobbs, and C. J. Taylor, "Survival of patients with chronic heart failure in the community: a systematic review and meta-analysis," European Journal of Heart Failure, vol. 21, no. 11, pp. 1306-1325, 2019.

[6] K. E. Freedland, M. W. Rich, and R. M. Carney, "Improving quality of life in heart failure," Current Cardiology Reports, vol. 23, no. 11, p. 159, 2021. 
[7] Q. Li, S. Liu, L. Zhang, Y. Li, X. Chen, and J. Hou, "Effects of Qi Li cardiac strenghten capsule combined with acupoint application on cardiopulmonary function," Journal of Hubei University of Chinese Medicine, vol. 23, no. 1, pp. 25-28, 2021.

[8] L. Li, "A clinical observation on treatment of CHF complicated with hyperuricemia with modified simiao pills and ear point pressing beans," Chinese Clinical Medicine, vol. 11, no. 20, pp. 34-36, 2019.

[9] X. Fan, J. Tian, X. Xu, W. Tian, and Y. Sun, "Clinical research of Yi Xin decoction with ear points in treating chronic contractive heart failure," Chinese Integrative Medicine, Chinese Journal of Integrative Medicine on Cardio-Cerebrovascular Disease, vol. 14, no. 19, pp. 2287-2289, 2016.

[10] G. Zhang, Y. Li, and Q. Lin, "Clinical research of yiqi huoxue lishui recipe in treating heart disease with qi deficiency, blood stasis and fluid retention syndrome," Chinese Journal of Integrative Medicine on Cardio-/Cerebrovascuiar Diseases, vol. 18, no. 19, pp. 3418-3152, 2020.

[11] X. Li, Y. Wu, Q. Li et al., "Randomize-controlled trial of yiqi huoxue lishui recipe adjuvant therapy in of CHF treatment," Journal of Traditional Chinese Medicine, vol. 60, no. 20, pp. 1741-1746, 2019.

[12] $\mathrm{H}$. Wu and G. Yuan, Integration of Chinese and Western Medicine on Treating Heart Failure, pp. 76-77, Tsinghua University Press, Beijing, China, 2008.

[13] S. A. Hunt, W. T. Abraham, M. H. Chin et al., "Focused update incorporated into the ACC/AHA 2005 Guidelines for the diagnosis and management of heart failure in adults: a report of the American college of cardiology foundation/ American heart association task force on practice guidelines: developed in collaboration with the international society for heart and lung transplantation," Circulation, vol. 119, no. 14, pp. e391-479, 2009.

[14] P. Qi and C. Zhang, "The latest healing guideline for heart failure diagnosis and treatment in ACC/AHA2009 (1)," Chinese Journal of Health Medicine, vol. 12, no. 4, pp. 323-325, 2010.

[15] X. Zheng, A Clinical Study on the Guidelines for Traditional Chinese Newly Investigated Drugs, pp. 77-85, China Medical and Clinical Science Press, Beijing, China, 2002.

[16] H. Wang and Y. Liang, "Diagnosis and treatment of Chinese people heart failure medical guidelines," Chinese Journal of Cardiology, vol. 46, no. 10, pp. 760-789, 2018.

[17] F. Wang and Z. Wang, Encyclopedia of Acupoint Special Therapy, Science and Technology Literature Press, Beijing, China, 1998.

[18] J. Yang, D. Wei, Q. Li, M. Chen, and Y. Zhang, “Application of tongue exercises of traditional Chinese medicine in improving the patients life quality with depression after chronic heart failure," Internal and External Therapy of Chinese Medicine, vol. 29, no. 5, pp. 3-5, 2020.

[19] W. Zha, Practical Acupoint Therapy Book, Jiangsu Science and Technology Press, Nanjing, China, 2004.

[20] J. Wan, Q. Lin, J. Su, H. Tan, and P. Li, "Evaluation on standard validity of life quality for chronic heart failure with integrated Chinese and Western medicine," Integrated Chinese and Western Medicine, vol. 36, no. 11, pp. 1300-1303, 2016.

[21] A. W. Chan, J. M. Tetzlaff, D. G. Altman et al., "SPIRIT 2013 statement: definition of standard protocol and clinical trials," Annals of Internal Medicine, vol. 158, no. 3, pp. 200-207, 2013.

[22] S. Li, "Implementation methods of common design schemes in clinical medical research," Chinese Journal of Practical Pediatrics, vol. 2008, no. 2, pp. 157-160, 2008.
[23] D. Tomasoni, M. Adamo, C. M. Lombardi, and M. Metra, "Highlights in heart failure," ESC Heart Failure, vol. 6, no. 6, pp. 1105-1127, 2019.

[24] P. Rossignol, A. F. Hernandez, S. D. Solomon, and F. Zannad, "Heart failure drug treatment," The Lancet, vol. 393, no. 10175, pp. 1034-1044, 2019.

[25] X. Li, J. Zhang, J. Huang et al., "A multicenter, randomized, double-blind, parallel-group, placebo-controlled study of the effects of qili qiangxin capsules in patients with chronic heart failure," Journal of the American College of Cardiology, vol. 62, no. 12, pp. 1065-1072, 2013.

[26] S. Fu, J. Zhang, F. Menniti-Ippolito et al., "Huangqi injection (a traditional Chinese patent medicine) for chronic heart failure: a systematic review," PLoS One, vol. 6, no. 5, Article ID e19604, 2011.

[27] J. Xue, Y. Xu, Y. Deng et al., "The Safety and efficiency of xin mai long injection with chronic heart failure: a multicenter randomized double-blind placebo-controlled trial," Journal of Alternative \& Complementary Medicine, vol. 25, no. 8, pp. 856-860, 2019.

[28] J. Jin, Study on the Intervention of Qishen Taohong Granule on Myocardial Remodeling in Mice with Heart Failure Based on mTOR/Autophagy Pathway, Beijing University of Chinese Medicine, Beijing, China, 2020.

[29] M. Toledano, J. W. Britton, A. McKeon et al., "Utility of an immunotherapy trial in evaluating patients with presumed autoimmune epilepsy," Neurology, vol. 82, no. 18, pp. 1578-1586, 2014.

[30] J. L. Li, C. Z. Liu, N. Zhang et al., "Neurological and psychological mechanisms of the specific and nonspecific effects of acupuncture on knee osteoarthritis: study protocol for a randomized, controlled, crossover trial," Trials, vol. 21, no. 1, p. 989, 2020. 